\title{
Revisión
}

\section{Inmunosenescencia, multimorbilidad, fragilidad y COVID-19}

\author{
William Arbey Gutiérrez Cortes $\mathbb{D}^{1}$ \\ ${ }^{1}$ Fundación Universitaria Navarra, Neiva (Huila), Colombia.
}

Cómo citar: Gutiérrez-Cortes W. Inmunosenescencia, multimorbilidad, fragilidad y covid-19. Rev. Colomb. Endocrinol. Diabet. Metab. 2021;8(1):e665. https://doi.org/10.53853/encr.8.1.665

Recibido: 09/Nov/2020

Aceptado: 23/Feb/2021

Publicado: $20 / \mathrm{Sep} / 2021$

\section{Resumen}

Objetivo: La pandemia por el covid-19 ha causado un aumento significativo en las hospitalizaciones y muertes por neumonía con enfermedad multiorgánica. Al tener en cuenta que las personas mayores han sido las más afectadas con la pandemia y que su alta mortalidad es debido a condiciones propias de la edad avanzada como son la fragilidad, la multimorbilidad y la inmunosenescencia, se propone describir brevemente estas variables y su relación con la mortalidad.

Materiales y métodos: Se realizó una búsqueda sistemática en bases de datos como PubMed y Scopus, con base en artículos de inmunosenescencia, multimorbilidad, fragilidad y covid-19 en el último año. Se utilizaron los términos MeSH de alta frecuencia, los cuales fueron: coronavirus, covid-19, frailty, immunosenescence, multimorbidity, sarcopenia. Se obtuvo un total de 500 artículos, se filtró por fecha, edad, lenguaje y tipo de artículo, obteniendo 50 artículos para la revisión.

Discusión: El covid-19 representa una gran amenaza para las personas mayores. Esto es particularmente cierto en personas con fragilidad y multimorbilidad.

Conclusión: Se reconoce que la pandemia requerirá esfuerzos permanentes en un marco de tiempo incierto, por lo que diferentes autores han llamado la atención en cuatro objetivos: primero, hacer más investigación clínica en personas mayores; segundo, vinculación de geriatras para la toma de decisiones en pacientes complejos; tercero, conocimiento de las necesidades de las poblaciones vulnerables; cuarto, enfatizar en la importancia de enfoques centrados en la persona que promulgen el respeto por la autonomía, la justicia y la beneficencia.

Palabras clave: coronavirus, covid-19, frailty, immunosenescence, multimorbidity, sarcopenia.

๑ Correspondencia: William Arbey Gutiérrez, Fundación Universitaria Navarra (Uninavarra), Calle 10 No. 6-41, Neiva (Huila), Colombia. Correo electrónico: willimedico@gmail.com 


\section{Immunosenescence, multimorbidity, frailty and COVID-19}

\section{Abstract}

Objective: The COVID-19 pandemic has caused a significant increase in hospitalizations and deaths from pneumonia with multi-organ disease. Taking into account that older persons have been the most affected by the pandemic and that their high mortality is due to conditions typical of advanced age such as frailty, Multimorbidity, and Immunosenescence, the author intends to briefly describe these variables and their relationship with mortality.

Methods: A systematic search was carried out in databases such as PubMed and Scopus, based on articles on immunosenescence, multimorbidity, frailty, and COVID-19 in the last year. The high-frequency MeSH terms were used, the terms were: Coronavirus, COVID-19, Frailty, Immunosenescence, Multimorbidity, Sarcopenia. A total of 500 articles were obtained, they were filtered by date, age, language, and type of article, obtaining 50 articles for review.

Discussion: COVID-19 poses a great threat to older persons. This is particularly true in people with frailty and multimorbidity.

Conclusions: It is recognized that the pandemic will require permanent efforts in an uncertain time frame, which is why different authors have drawn attention to four objectives: first, to do more clinical research in older persons, second, to link geriatricians for decision making, third, knowledge of the needs of vulnerable populations, and fourth emphasize the importance of person-centered approaches that promote respect for autonomy, justice, and beneficence.
Destacados

El $80 \%$ de la mortalidad por COVID-19 en Colombia se presentó en personas mayores.

La alta mortalidad se debe a la edad avanzada, la fragilidad y la multimorbilidad.

La evidencia disponible sugiere la importancia de involucrar a geriatras en el manejo de estos pacientes.

Keywords: Coronavirus, covid-19, frailty, immunosenescence, multimorbidity, sarcopenia.

La pandemia por el covid-19 ha causado un aumento significativo en las hospitalizaciones por neumonía con enfermedad multiorgánica. La infección por SARS-CoV-2 (severe acute respiratory syndrome coronavirus 2) puede ser asintomática o puede causar un amplio espectro de síntomas y sepsis potencialmente mortal (1).

Desde el primer caso en Colombia que se documentó el 6 de marzo del 2020, se inició una implementación de intervenciones para disminuir la tasa de contagios, reducir el índice reproductivo efectivo (Rt) y en consecuencia la mortalidad. Cuando se inició la cuarentena el 22 de marzo del 2020 se planteaba un escenario con un Rt de 2,28, con evidencia internacional que promediaba un Rt de 2,28 a 5,27; dicha intervención que el Gobierno presentó con el nombre de aislamiento preventivo obligatorio fue efectiva para lograr el distanciamiento físico que llevó a una reducción significativa de casos, logrando para el 20 de julio tener un promedio nacional de Rt en $1,17(2,3)$.

Para el 22 de octubre nos acercábamos al millón de casos de covid-19 (981.700) con una mortalidad de 29.464 personas (4). La mortalidad en Bogotá, Caldas, Valle del Cauca y Antioquia es cercana al $80 \%$ en mayores de 60 años (ver tabla I). Al tener en cuenta que las personas mayores han sido las más afectadas con la pandemia y que su alta mortalidad es debida a condiciones propias de la edad avanzada, como son la fragilidad, la multimorbilidad (5-7) y la inmunosenescencia $(8,9)$ (ver figura 1$)$, ello condiciona a este grupo poblacional a peores desenlaces, por lo que el autor se propone describir brevemente estas variables mediante una revisión actualizada de la literatura. 


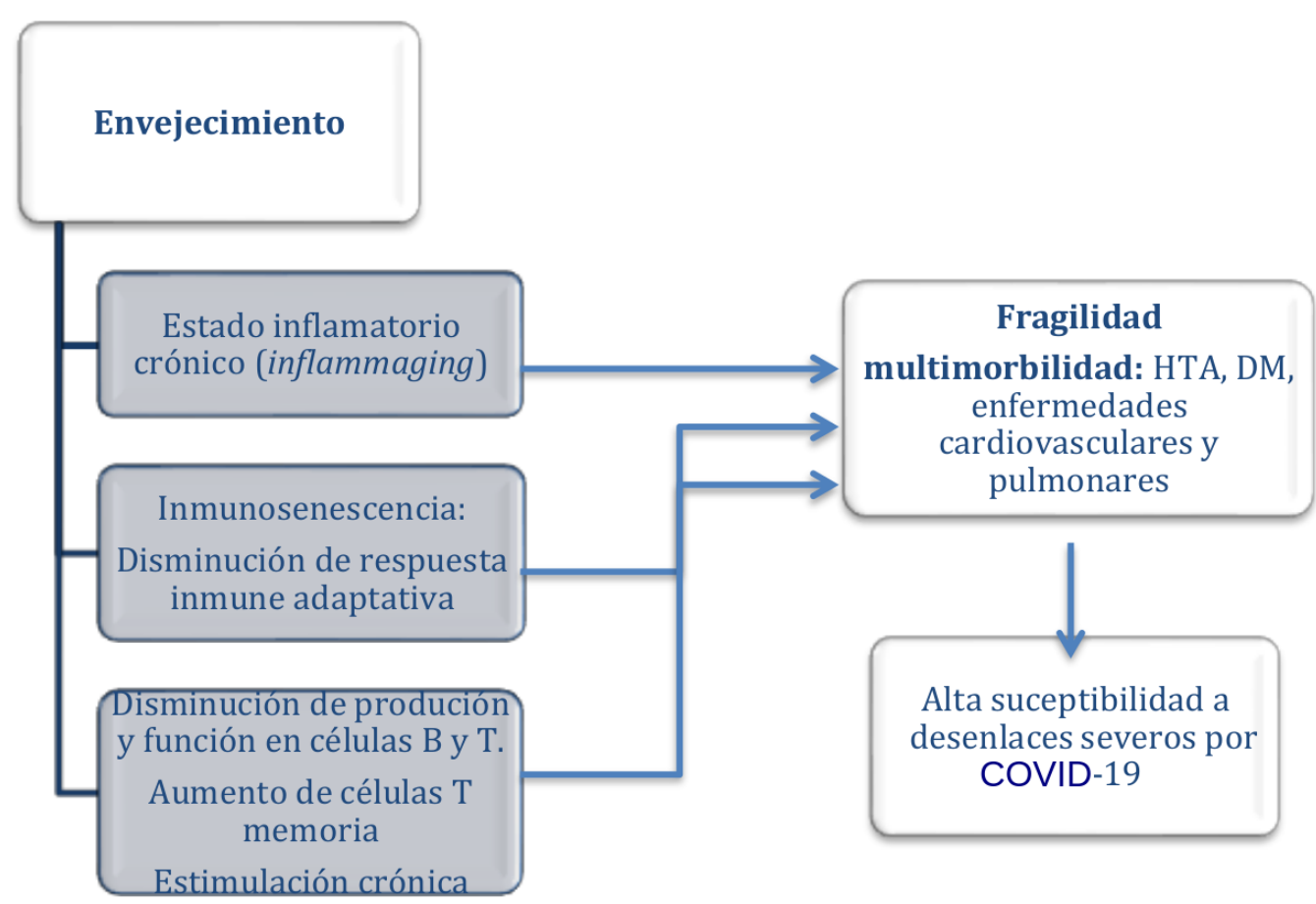

Figura 1. Ciclo de envejecimiento e infección por SARS-COV-2

Fuente: los autores.

Tabla I. Mortalidad en mayores de 60 años por regiones (Datos del 06/03/2020 a 22/10/2020)

\begin{tabular}{|c|c|c|c|}
\hline Región & $\begin{array}{c}\text { Población mayor } \\
\text { de 60 años }\end{array}$ & $\begin{array}{c}\text { Mortalidad global } \\
\text { covid-19 }\end{array}$ & $\begin{array}{c}\text { Mortalidad de mayores } \\
60 \text { años (\%) }\end{array}$ \\
\hline Bogotá & 995.029 & 7.368 & $5.591(75 \%)$ \\
\hline Caldas & 150.425 & 220 & $182(82 \%)$ \\
\hline Valle del Cauca & 609.176 & 2.453 & $1.972(80 \%)$ \\
\hline Antioquia & 818.096 & 2.911 & $2.352(80 \%)$ \\
\hline
\end{tabular}

Fuente: Instituto Nacional de Salud (10).

\section{Materiales y métodos}

Se realizó una búsqueda sistemática en bases de datos como PubMed y Scopus, con base en artículos de inmunosenescencia, multimorbilidad, fragilidad y covid-19 en el último año. Como herramienta se utilizaron los términos MeSH de alta frecuencia y de esta manera, mediante la revisión de cada uno, se logró construir una revisión en relación con dichas variables y el incremento de la mortalidad. Los términos utilizados fueron: coronavirus, covid-19, frailty, immunosenescence, multimorbidity y sarcopenia, se obtuvo un total de 500 artículos, se filtró por fecha, edad, lenguaje y tipo de artículo, obteniendo 50 artículos para la presente revisión. 
Respuesta inmune a la infección por covid-19

El covid-19 se puede dividir en tres fases: asintomática con o sin virus detectable, sintomática no grave con presencia de virus y sintomática respiratoria grave con alta carga viral (11). Una pregunta no resuelta es por qué algunas personas desarrollan enfermedad grave y otros no. Los aspectos basados en la respuesta inmune no son suficientes para explicarlo, pero ayudarán a entender el comportamiento de este nuevo patógeno.

Los linfocitos $T(L T)$, linfocitos B (LB) y las asesinas naturales (NK) tienen un papel importante en mantener el sistema inmune. En la infección por SARS-CoV-2, los estudios demuestran que hay una marcada linfopenia (12). En la sangre de un paciente se encontró una linfopenia de LT CD4+ y CD8+, sin embargo, ambas poblaciones presentaban un estatus hiperactivado con altas proporciones de CD4+HLA-DR+ $(3,5 \%)$ y CD8+CD38+ (39,4\%). Además, se encontraron proporciones elevadas de LT proinflamatorios CD4+CCR6+ y LT CD8+ con altas cantidades de gránulos citotóxicos. Estas poblaciones linfocitarias podrían explicar parcialmente el grave daño al sistema inmune (12).

En otros pacientes con infección grave también se han observado: linfopenia, mayor relación neutrófilos/linfocitos, menor cantidad de monocitos, eosinófilos y basófilos, en comparación con los pacientes sin síntomas de la enfermedad (13). Dentro de los grupos celulares más afectados están los LT (CD4+ y CD8+), los cuales estuvieron por debajo de los valores normales y fue más evidente en el caso de los LT CD4+ de pacientes graves. Estos resultados coinciden con los de Wang et al., ya que son cercanos del $30 \%$ de pacientes con enfermedad grave (12). Por otro lado, los LT CD4+CD45RA+ (vírgenes) se incrementan mientras que los LT CD4+CD45RO+ (memoria) disminuyen. El nivel de activación solo disminuye en los LT CD8+CD28+ junto con una disminución de $\mathrm{LT} \mathrm{CD} 4+$ reguladores vírgenes (CD45RA+CD127Low+) e inducidos (CD45RA+CD127Low+).

Estos datos también sugieren que el sistema inmune está desregulado durante el curso de la enfermedad por SARS-CoV-2 y es más crítica cuando el paciente tiene comorbilidades (14).

\section{Estado inflamatorio crónico (infla- mmaging) e inmunosenescencia}

La alteración más notable es que las células $T$ y $B$ de memoria reemplazan a las células $\mathrm{T}$ y $\mathrm{B}$ vírgenes (15). El reemplazo de células inmunes vírgenes disminuye las respuestas de adaptación a nuevos antígenos como el SARS-CoV-2. Por otro lado, la protección inmunitaria de las células $T$ de memoria frente a la reinfección viral con patógenos conocidos suele aumentar con la edad (16).

Las respuestas inmunes se dividen a grandes rasgos en (a) respuestas innatas, transportadas principalmente por neutrófilos, macrófagos y células NK que reaccionan al patógeno de forma rápida e inespecífica, y (b) respuestas adaptativas, transportadas por linfocitos $T$ y $B$ que se retrasan, son más lentas y específicas (por ejemplo: expansión clonal específica de antígeno de linfocitos $T$ y $B$ y producción de anticuerpos por linfocitos B) (16). 
En las personas mayores las respuestas inmunitarias al SARS-CoV-1 y 2 están "atrapadas en la inmunidad innata", con una progresión insuficiente hacia la inmunidad adaptativa (17); sin embargo, la disminución de la respuesta adaptativa, como la producción de anticuerpos, juega un papel pequeño en la mortalidad por covid-19. Es la inmunidad innata hiperfuncional, la hiperinflamación, la tormenta de citocinas y la hipercoagulación las que conducen a la insuficiencia orgánica y la muerte (18).

En las personas mayores, las células inmunitarias innatas se encuentran en un estado de activación sostenida, produciendo citocinas proinflamatorias (19). El aumento de la actividad proinflamatoria por parte del sistema inmunológico innato, especialmente por los monocitos/macrófagos, es un estado de alerta e hiperreactividad sobre el costo de posibles enfermedades inflamatorias relacionadas con la edad, mientras que algunas funciones disminuyen, otras aumentan.

De acuerdo con el concepto de envejecimiento inflamatorio, el sistema inmunológico innato supera al sistema inmunológico adaptativo en el envejecimiento. Las relaciones causa-efecto son bidireccionales: la inmunosenescencia (es decir, una disminución de la respuesta adaptativa) es una causa y una consecuencia del envejecimiento inflamatorio $(20,21)$ (ver figura 1).

\section{Multimorbilidad}

A finales de julio, el Ministerio de Salud y Protección Social dio a conocer el primer análisis de comorbilidades y otros factores relacionados con los fallecimientos en el país por Covid-19. En el documento se analizaron 123.000 casos a partir de historias clínicas que reveló el riesgo de mortalidad por comorbilidades (22).

Se hallaron ocho condiciones que configuran mayor riesgo de morir. La edad es el factor de mayor peso en los desenlaces fatales. De hecho, se encontró que las personas mayores de 60 años tienen 19 veces más riesgo de morir. Lo anterior surge al verificar que alrededor de los 60 años hubo 18.616 casos y 3.282 fallecidos. La estimación del riesgo de muerte fue exactamente de 18,86. El estudio también referencia que en el grupo de 70 años o más, el riesgo disminuye un poco 17,59 , basado en la observación de 8.691 casos y 2.238 muertes.

De entrada, el estudio evidencia que tener cualquier comorbilidad aumenta el riesgo de muerte en 5,94 veces frente a quienes no la padecen, pero en una mirada por patologías específicas, la enfermedad renal crónica configura un factor importante relacionado con las muertes por covid-19, al apreciarse que quienes están en diálisis tienen 8,55 veces más probabilidades de fallecer (22). A continuación está la diabetes, que con 860 fallecidos, producto de 5.491 contagios, aumenta en 5,9 veces el riesgo de morir por covid-19. Por otra parte, se encontró que el riesgo de morir por efectos del nuevo coronavirus en pacientes con cáncer es de 4,5 veces mayor que el de una persona sana (ver tabla II).

\section{Fragilidad}

Es un síndrome de reducción de reserva física, fisiológica y cognitiva, originado en múltiples factores que afectan negativamente la reserva de homeostasis individual, con la consecuente 
vulnerabilidad a estresores externos, predisponiendo a las personas a un alto riesgo de desenlaces adversos como otros síndromes geriátricos, hospitalización, institucionalización, discapacidad y muerte $(23,24)$.

Tabla II. Comorbilidades asociadas a mortalidad por COVID-19

\begin{tabular}{|c|c|c|c|}
\hline Factor medio & Número de casos & Fallecidos & $\begin{array}{c}\text { Estimación del riesgo } \\
\text { de mortalidad }\end{array}$ \\
\hline Mayores de 60 años & 18.616 & 3.282 & 18,86 \\
\hline Mayores de 70 años & 8.691 & 2.238 & 17,59 \\
\hline HTA & 14.739 & 1.945 & 6,43 \\
\hline DM & 5.491 & 860 & 5,91 \\
\hline
\end{tabular}

Fuente: El Tiempo, 2020 (22).

En investigación y la práctica clínica existen múltiples formas de identificar la fragilidad, desde instrumentos de tamizaje rápidos y cortos como la escala de FRAIL (25), el original fenotipo de Fried (26), hasta las mediciones más completas, sofisticadas y que requieren mayor tiempo de aplicación (índice de Roockwood Mitnitski) (27).

Independiente del método utilizado, es necesario identificar de modo rutinario a las personas mayores frágiles o en riesgo de padecerla (prefrágiles) en la búsqueda de predecir resultados y respuesta a tratamientos potenciales (28). Especialmente en personas mayores agudamente enfermos y con necesidad de cuidado crítico, como son los pacientes con covid-19. Entre los instrumentos validados para evaluar fragilidad en pacientes críticos se encuentra la escala clínica de fragilidad (CFS, sigla en inglés para clinical frailty scale) (29). Esta escala de uso ordinal de puntaje de 1 (robustos) a 9 (enfermos terminales) de los cuales CFS de 5 a 8 se consideran frágiles, incluye la evaluación de dominios físicos, cognitivos, comorbilidad y discapacidad, reconociendo el juicio clínico y la importancia de la funcionalidad (30).

La prevalencia de la fragilidad es variable y dependerá de factores como el método de diagnóstico, el tipo de paciente y el escenario donde sea evaluada. Se estima que un $15 \%$ de las personas residentes en comunidad en Estados Unidos son frágiles según el fenotipo de Fried (31), mientras que a nivel mundial puede variar en un rango de $3,5 \%$ a $27,3 \%$ (32). En Colombia, la prevalencia de fragilidad oscila entre 9,4\% y $12,1 \%(33,34)$.

Específicamente, se reconoce un aumento en la mortalidad a doce meses por cada punto de aumento de la CFS, es decir, estar en CFS de 1 (es decir ser una persona mayor robusta) estuvo asociado con una tasa de supervivencia a un año del $90 \%$, una escala de CFS de 5 está asociada con $50 \%$ de supervivencia y aquellos con CFS de 6 o 7 tienen un $35 \%$ de supervivencia (35).

Al categorizar a los pacientes de acuerdo con el índice de fragilidad de Rockwood y Miritski como robustos (IF $<0,25)$, pre frágiles (IF $0,25-0,4)$ y frágiles (> 
$0,4)$, hay un aumento de la mortalidad a seis meses a medida que aumenta el índice de fragilidad de 55,9\%, 70,3\% y $84,6 \%$ respectivamente $(35,36)$. No obstante, existen otros desenlaces como el uso de ventilación mecánica o terapia con vasoactivos, en los cuales no se ha demostrado evidencia significativa. Es fundamental tener esta evidencia en mente, pues se espera que la mayor proporción de adultos mayores con covid-19 tendrá manifestaciones de severidad de la enfermedad, por lo tanto, se debe conocer la condición de fragilidad de los pacientes incluso antes de enfermar agudamente, inclusive guías como NICE (National Institute for Health and Care Excellence) han sugerido el uso de la CFS en la toma de decisiones de ingreso a cuidado intensivo para pacientes positivos para covid-19 (37).

Debido a la naturaleza reversible de las transiciones de la fragilidad y que no es un sinónimo de fin de vida, se recomienda que sea tomado como un marcador conjunto a la multimorbilidad previa y a la severidad de la enfermedad, en la toma de decisiones terapéuticas en mayores con covid-19 (38).

\section{Sarcopenia}

La sarcopenia es un término derivado del griego sarx (carne) y penia (pérdida), el cual fue acuñado por primera vez por Irwin Rosenberg en 1989 para referirse a la pérdida de masa muscular con la edad. Desde ese entonces y hasta nuestra época, este término ha evolucionado y la patología se ha tenido en cuenta, al relacionar la masa muscular con su función y los cambios que se presentan con el envejecimiento (39-41). Según el Grupo de Trabajo sobre Sarcopenia en las Personas Mayores (Ewgsop), esta se define como baja masa muscular en combinación con un bajo rendimiento físico o debilidad en la fuerza muscular $(42,43)$.

El covid-19 provoca anorexia, pérdida de peso e hipoalbuminemia, además se han identificado niveles séricos elevados de PCR y citocinas proinflamatorias (IL6-IL10, FNT), fenómeno que coexiste con el inflammaging y que agrava la pérdida muscular causante de sarcopenia.

En personas mayores, la necesidad de aislamiento físico durante la pandemia ha llevado a una disminución de la actividad física diaria que acelera la pérdida de fuerza y la función muscular. En los pacientes diagnosticados con covid-19 es probable que tengan de dos o tres semanas de limitación funcional, lo que resulta en sarcopenia secundaria (44). Existe evidencia de que los pacientes con covid-19 severo necesitan una terapia de ejercicio prolongada para prevenir o revertir la discapacidad generada por la infección por el nuevo coronavirus $(45,46)$.

\section{Discusión}

El proceso de inmunosenescencia es un proceso dinámico en el que interfieren múltiples factores a lo largo de la vida, se han descrito factores intrínsecos como lo son los genéticos, la reducción de la masa muscular, el resecamiento de las mucosas, el deterioro de la piel y extrínsecos como lo son la alimentación, la actividad física, los tratamientos farmacológicos, la fragilidad, las comorbilidades y el estrés, por lo que situaciones como la malnutrición, el aislamiento, el estrés, la depresión, el sedentarismo y la inmovilidad contribuyen a la desregulación de la función 


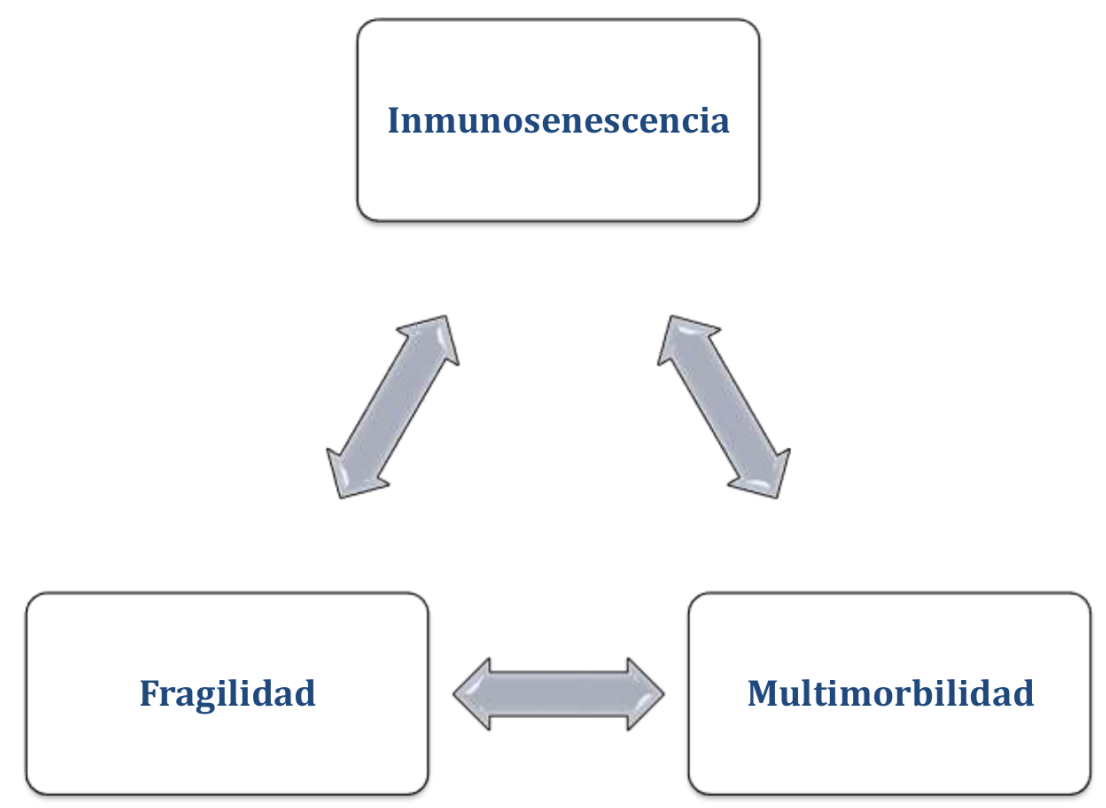

Figura 2. Condiciones que aumentan la mortalidad en personas mayores por COVID-19

Fuente: los autores.

inmune (9). Estos factores pueden modular el inicio temprano o el desarrollo de la inmunosenescencia, generando una disminución funcional progresiva en la mayoría de las poblaciones celulares que hacen parte del sistema inmune, alterando su activación, por lo que las intervenciones desde la atención primaria y promoción en salud son de gran importancia para promover un envejecimiento saludable.

El covid-19 representa una gran amenaza para las personas mayores (figura 2), mucho más en pacientes con fragilidad y multimorbilidad por lo que es importante el inicio de la vacunación y su aplicación masiva en dicha población (47).

En Colombia a la fecha no existe una atención diferencial en personas mayores que involucre determinantes sociales y un enfoque transversal y multidisciplinar que busque reducir la carga de morbilidad e involucre un abordaje desde la atención primaria hasta el nivel hospitalario coordinado y liderado por geriatras. La experiencia internacional sugiere la importancia de involucrar a especialistas en geriatría para la correcta evaluación y manejo de pacientes con covid-19. Estos pacientes tienen alta carga de comorbilidad, polifarmacia y dependencia funcional, por lo que necesitan de médicos entrenados para lidiar con tales condiciones $(47,48)$.

\section{Conclusión}

Se reconoce que la pandemia requerirá esfuerzos permanentes en un marco de tiempo incierto, por lo que diferentes autores han llamado la atención en cuatro objetivos: primero, hacer más investigación clínica que incluya todas las edades; segundo, vinculación de geriatras para la toma de decisiones institucionales en pacientes complejos; tercero, conocimiento de las necesidades de las poblaciones vulnerables; cuarto, enfatizar en la 
importancia de enfoques centrados en la persona que promulguen el respeto por la autonomía, la justicia y la beneficencia (49-51).

\section{Agradecimientos}

A mi familia por su acompañamiento.

\section{Conflicto de intereses}

El autor declara que no existen conflictos de interés.

\section{Referencias}

[1] Wiersinga WJ, Rhodes A, Cheng AC, Peacock SJ, Prescott HC. Pathophysiology, Transmission, Diagnosis, and Treatment of Coronavirus Disease 2019 (COVID-19). A Review. JAMA. 2020 ag. 25;324(8):782-93. doi: https://doi.org/10.1001/jama.2020. $12839 \uparrow$ Ver página 2

[2] Roselli D. Covid-19 en Colombia: los primeros 90 días. Acta Neurol Colomb. 2020 jun.;36(2):1-6. doi: https://doi. org/10.22379/24224022287 个Ver página 2

[3] Gutiérrez-Cortes WA. Papel del geriatra colombiano ante la pandemia por COVID 19. Rev Esp Geriatr Gerontol. 2020;55(6):358-9. doi: https://doi. org/10.1016/j.regg.2020.06.010 个Ver página 2

[4] Instituto Nacional de Salud [sitio virtual]. Boletín Covid 19 En Colombia. Bogotá: INS; [citado $X X X X X X]$. Disponible en: https:

\section{//www.ins.gov.co/Noticias/Paginas/} Coronavirus.aspx $\uparrow$ Ver página 2

[5] Huang C, Wang $Y$, Li $X$, Ren L, Zhao J, $\mathrm{Hu} \mathrm{Y}$, et al. Clinical features of patientsinfected with 2019 novel coronavirus in Wuhan, China. Lancet. 2020 febr. 15;395(10223):497-506. doi: https://doi.org/10.1016/ S0140-6736(20)30183-5 个Ver página 2

[6] Hewitt J, Carter B, Vilches-Moraga A, Quinn TJ, Braude P, Verduri A, et al. The effect of frailty on survival in patients with COVID-19 (COPE): a multicentre, European, observational cohort study. Lancet Public Health. 2020 ag.;5(8):e444-e451. doi: https://doi. org/10 . 1016/S2468-2667 (20)30146-8 个Ver página 2

[7] Wang D, Hu B, Hu C, Zhu F, Liu $X$, Zhang $J$, et al. Characteristics of 138 Hospitalized Patients With 2019 Novel Coronavirus-Infected Pneumonia in Wuhan, China. JAMA. 2020 mzo. 17;323(11):1061-9. doi: https: //doi.org/10.1001/jama.2020.1585 个Ver página 2

[8] Vellas C, Delobel P, de Souto-Barreto P, lzopet J. COVID-19, virology and geroscience: a perspective. J Nutr Health Aging. 2020;24(7):685-691. doi: https://doi.org/10.1007/ s12603-020-1416-2 个Ver página 2

[9] Grupo de Inmunología y Medicina Traslacional. La inmunosenescencia: orientaciones y propuestas para un envejecimiento saludable, información básica para personal del sector salud. Bogotá, Colombia: Facultad de Medicina, 
Universidad Nacional de Colombia; 2020. $\uparrow$ Ver páginas 2,8

[10] Instituto Nacional de Salud. Boletín Covid 19 En Colombia (22 Octubre del 2020). Bogotá: INS. https://www.ins.gov.co/Noticias/ Paginas/Coronavirus.aspx. $\quad \uparrow$ Ver páginas 3

[11] Lozada-Requena I, Núñez-Ponce C. COVID-19: respuesta inmune y perspectivas terapéuticas. Rev Peru Med Exp Salud Pública. 2020;37(2):312-9. doi: https://doi.org/10.17843/ rpmesp.2020.372.5490 $\uparrow$ Ver página 4

[12] Wang F, Nie J, Wang H, Qiu Z, Yong $X$, Liping $D$, et al. Characteristics of peripheral lymphocyte subset alteration in COVID-19 pneumonia. J Infect Dis. 2020 my. 11;221(11):1762-9. doi: https://doi.org/10.1093/infdis/ jiaa150. $\uparrow$ Ver página 4

[13] Xu Z, Shi L, Wang Y, Zhang J, Huang $L$, Zhang $C$, et al. Pathological findings of COVID-19 associated with acute respiratory distress syndrome. Lancet Respir Med. 2020;2600(20):19-21. doi: https://doi.org/10.1016/ S2213-2600(20)30076-X 个Ver página 4

[14] Qin C, Zhou L, Hu Z, Zhang S, Yang $\mathrm{S}$, Tao $Y$, et al. Dysregulation of immune response in patients with COVID-19 in Wuhan, China. Clin Infect Dis. 2020 jul. 28;71(15):762-768. doi: https: //doi.org/10.1093/cid/ciaa248. $\uparrow$ Ver página 4

[15] de la Rica $R$, Borges $M$, Gonzalez-Freire M. COVID-19: in the eye of the cytokine storm. Front Immunol. 2020 sept. 24;11:558898. doi: https: //doi.org/10.3389/fimmu.2020.558898 个Ver página 4

[16] Bencivenga L, Rengo G, Varricchi G. Elderly at time of Coronavirus disease 2019 (COVID-19): possible role of immunosenescence and malnutrition. Geroscience. 2020 ag.;42(4):1089-92. doi: https://doi.org/10.1007/ s11357-020-00218-9 Ver página 4

[17] Napoli C, Tritto I, Mansueto $G$, Coscioni E, Ambrosio G. Immunosenescence exacerbates the COVID-19. Arch Gerontol Geriatr. 2020 sept.;90:104174. doi: https ://doi .org/ 10.1016/j.archger.2020.104174 $\uparrow$ Ver página 5

[18] Fuentes $E$, Fuentes $M$, Alarcón $M$, Palomo I. Immune System Dysfunction in the Elderly. An Acad Bras Cienc. 2017 mzo;89(1):285-99. doi: https://doi. org/10.1590/0001-3765201720160487 个Ver página 5

[19] Kadambari $S$, Klenerman $P$, Pollard AJ. Why the elderly appear to be more severely affected by COVID-19: The potential role of Immunosenescence and CMV. Rev Med Virol. 2020 sept.;30(5):e2144. doi: https://doi.org/10.1002/rmv.2144 $\uparrow$ Ver página 5

[20] Cunha LL, Perazzio SF, Azzi J, Cravedi P, Riella LV. Remodeling of the immune response with aging: immunosenescence and its potential impact on COVID-19immune response. Front Immunol. 2020 ag. 7;11:1748. doi: https://doi.org/10.3389/fimmu. 2020. $01748 \uparrow$ Ver página 5 
[21] Chen J, Kelley WJ, Goldstein DR. Role of aging and the immune response to respiratory viral infections: potential implications for COVID-19. J Immunol. 2020 jul. 15;205(2):313-320. doi: https : //doi.org/10.4049/jimmunol.2000380 个Ver página 5

[22] El Tiempo [Internet]. Bogotá: El Tiempo; 2020 jul. 24. La edad y estas enfermedades aumentan el riesgo de morir por covid-19. [citado XXXXX]. Disponible en: https://www.eltiempo.com/salud/ coronavirus-colombia-hoy-estas-son\ - las-enfermedades-ligadas-a-las-mu\ ertes-por-covid-19-518600 $\quad \uparrow$ Ver páginas 5, 6

[23] Belloni G, Cesari M. Frailty and intrinsic capacity: two distinct but related constructs. Front Med (Lausanne). 2019 jun. 18;6:133. doi: https: //doi.org/10.3389/fmed.2019.00133 个Ver página 6

[24] Vermeiren S, Vella-Azzopardi R, Beckwée $D$, Habbig AK, Scafoglieri $A$, Jansen $B$, et al. Frailty and the prediction of negative health outcomes: a meta-analysis. J Am Med Dir Assoc. 2016;17:1163. doi: https://doi.org/ $10.1016 / j \cdot j a m d a .2016 .09 .010 \quad \uparrow V e r$ página 6

[25] Morley JE, Vellas B, van Kan GA, Anker SD, Bauer JM, Bernabei R, et al. Frailty consensus: a call to action. J Am Med Dir Assoc. 2013 jun.;14(6):392-7. doi: https://doi.org/10.1016/j.jamda. 2013.03.022 个Ver página 6

[26] Fried LP, Tangen CM, Walston J, Newman AB, Hirsch C, Gottdiener J, et al. Frailty in older adults: evidence for a phenotype. J Gerontol A Biol SciMed Sci. 2001;56:M146-56. doi: https: //doi.org/10.1093/gerona/56.3.m146 个Ver página 6

[27] Rockwood $K$, Song $X$, MacKnight C, Bergman $H$, Hogan DB, McDowell I, et al. Aglobal clinical measure of fitness and frailty in elderly people. CMAJ Can Med Assoc J J Assoc Medicale Can. 2005;173:489-95. doi: https://doi.org/10.1503/cmaj.050051 个Ver página 6

[28] Dent E, Kowal P, Hoogendijk EO. Frailty measurement in research and clinical practice: a review. Eur J Intern Med. 2016;31:3-10. doi: https://doi. org/10.1016/j.ejim.2016.03.007 个Ver página 6

[29] Shears $M$, Takaoka A, Rochwerg $B$, Bagshaw SM, Johnstone J, Holding $A$, et al. Assessing frailty in the intensive care unit: A reliability and validity study. J Crit Care. 2018 jun.;45:197-203. doi: https://doi.org/10.1016/j.jcrc. 2018.02.004 $\uparrow$ Ver página 6

[30] Hope AA, Hsieh SJ, Petti A, Hurtado-Sbordoni $M$, Verghese J, Gong MN. Assessing the Usefulness and Validity of Frailty Markers in Critically III Adults. Ann Am Thorac Soc. 2017 jun.;14(6):952-959. doi: https://doi. org/10 . 1513/AnnalsATS . 201607-5380C 个Ver página 6

[31] Bandeen-Roche K, Seplaki CL, Huang J, Buta B, Kalyani RR, Varadhan R, et al. Frailty in older adults: a nationally representative profile in the United States. J Gerontol A Biol Sci Med Sci. 
2015 nov.;70(11):1427-34. doi: https: //doi.org/10.1093/gerona/glv133 个Ver página 6

[32] Walston J, Buta B, Xue QL. Frailty screening and interventions: considerations for clinical practice. Clin Geriatr Med. 2018 febr.;34(1):25-38. doi: https://doi.org/10.1016/j.cger. 2017.09.004 个Ver página 6

[33] Gómez-Montes JF, Curcio-Borrero $\mathrm{CL}$, Henao GM. Fragilidad en ancianos colombianos. Rev Médica Sanitas. 2012;15(4):8-16. $\uparrow$ Ver página 6

[34] Muscedere J, Waters B, Varambally A, Bagshaw SM, Boyd JG, Maslove D, et al. The impact of frailty on intensive care unit outcomes: a systematic review andmeta-analysis. Intensive Care Med. 2017 ag.;43(8):1105-22. doi: https:// doi .org/10.1007/s00134-017-4867-0

个Ver página 6

[35] Brummel NE, Bell SP, Girard TD, Pandharipande PP, Jackson JC, Morandi $A$, et al. Frailty and subsequent disability and mortality among patients with critical illness. Am J Respir Crit Care Med. 2017 jul. 1;196(1):64-72. doi: https:// doi.org/10.1164/rccm.201605-09390C $\uparrow$ Ver página 6,7

[36] Kizilarslanoglu MC, Civelek R, Kilic MK, Sumer F, Varan HD, Kara O, et al. Is frailty a prognostic factor for critically ill elderly patients? Aging Clin Exp Res. 2017 abr.;29(2):247-255. doi: https:// doi .org/10.1007/s40520-016-0557-y 个Ver página 7

[37] National Institute for Health and Care Excellence [Internet]. Reino Unido: NICE; 2020. COVID-19 rapid guideline:

Volumen 8, número 1 de 2021 critical care in adults [citado 2020 abr. 11]. Disponible en: https://www. nice. org. uk/guidance/ng159/resources \ /critical-care-admission-algorithm \ -pdf-87089488934 ^Ver página 7

[38] Hewitt J, Carter B, Vilches-Moraga $A$, Quinn TJ, Braude P, Verduri $A$, et al. The effect of frailty on survival in patients with COVID-19 (COPE): a multicentre, European, observational cohort study. Lancet Public Heal [Internet]. 2020;0(0):1-8. Disponible en: https://linkinghub.elsevier.com/ retrieve/pii/S2468266720301468. $\uparrow$ Ver página 7

[39] Bauer J, Morley JE, Schols AM, Ferrucci L, Cruz-Jentoft AJ, Dent E, et al. Sarcopenia: a time for action. An SCWD position paper. J Cachexia Sarcopenia Muscle. 2019;10:956-61. doi: https://doi.org/10.1002/jcsm. 12483 $\uparrow$ Ver página 7

[40] Dent E, Morley JE, Cruz-Jentoft AJ, Arai H, Kritchevsky SB, Guralnik $J$, et al. International clinical practice guidelines for sarcopenia (ICFSR): screening,diagnosis and management. J Nutr Health Aging. 2018;22:1148-61. doi: https://doi.org/10.1007/ s12603-018-1139-9 $\uparrow$ Ver página 7

[41] Wandrag $L$, Siervo $M$, Riley $H L$, Khosravi M, Fernandez BO, Leckstrom $C A$, et al. Dose hypoxia play a role in the development of sarcopenia in humans? Mechanistic insights from the Caudwell Xtreme Everest expedition. Redox Biol. 2017;13:60-8. doi: https://doi.org/ 10.1016/j.redox.2017.05.004 $\uparrow$ Ver página 7

http://revistaendocrino.org/index.php/rcedm 
[42] Gutiérrez W, Martínez F, Olaya L. Sarcopenia, una patología nueva que impacta a la vejez. Rev Colomb Endocrinol Diabetes Metabol. 2018 febr.;5(1):28-36. doi: https: //doi.org/10.53853/encr.5.1.339 $\uparrow$ Ver página 7

[43] Izquierdo $M$, Rodriguez-Mañas $L$, Sinclair AJ. Editorial: what is new in exercise regimes for frail older people how does the Erasmus Vivifrail project take us forward? J Nutr Health Aging. 2016;20:736-7. doi: https://doi. org/10.1007/s12603-016-0702-5 $\uparrow$ Ver página 7

[44] Izquierdo $M$, Morley JE, Lucia A. Exercise in people over 85. BMJ. 2020 febr. 5;368:m402. doi: https://doi.org/10.1136/bmj.m402 $\uparrow$ Ver página 7

[45] Li J. Effect and enlightenment of rehabilitation medicine in COVID-19 management. Eur J Phys Rehabil Med 2020. (Epub ahead of print). doi: https://doi.org/10.23736/ S1973-9087.20.06292-9 个Ver página 7

[46] Morley JE, Kalantar-Zadeh K, Anker SD. COVID-19: a major cause of cachexia and sarcopenia? J Cachexia Sarcopenia Muscle. 2020 ag.i11(4):863-5. doi: https://doi.org/10.1002/jcsm.12589 $\uparrow$ Ver página 7
[47] Morley JE, Vellas B. Editorial: Covid-19 and older adults. J Nutr Health Aging. 2020;24(4):364-5. doi: https:// doi.org/10.1007/s12603-020-1349-9 个Ver página 8

[48] Haffajee RL, Mello MM. Thinking globally, acting locallydthe U.S. response to COVID-19. N Engl J Med. 2020 my. 28;382(22):e75. doi: https: //doi.org/10.1056/NEJMp2006740 个Ver página 8

[49] Landi F, Barillaro C, Bellieni A, Brandi $V$, Carfi A, D'Angelo $M$, et al. The new challenge of geriatrics: saving frail older people from the SARS-CoV-2 pandemic infection. J Nutr Health Aging. 2020;24(5):466-470. doi: https://doi. org/10.1007/s12603-020-1356-x $\uparrow V e r$ página 9

[50] Lloyd-Sherlock PG, Kalache A, McKee $M$, Derbyshire J, Geffen L, Casas FG. WHO must prioritise the needs of older people in its response to the covid-19 pandemic. BMJ. 2020 mzo. 23;368:m1164. doi: https://doi.org/10.1136/bmj.m1164 $\uparrow$ Ver página 9

[51] Colenda CC, Reynolds CF, Applegate WB, Sloane PD, Zimmerman S, Newman $A B$, et al. Covid-19 pandemic and ageism: a call for humanitarian care. Am J Geriatr Psychiatry. 2020 ag.;28(8):805-7. doi: https://doi.org/10.1016/j.jagp. 2020.04.005 $\uparrow$ Ver página 9 